\title{
Current Concepts Surrounding Project Management for Complex Performance Improvement Initiatives
}

\author{
Frederick van Pelt • Alejandro Kohn Tuli
}

Published online: 8 July 2014

(c) Springer Science+Business Media New York 2014

\begin{abstract}
Hospitals are complex organizations that utilize highly trained, autonomously performing clinical professionals. Hospitals are now borrowing management practices from other industries. Most facilities have turned toward internal process improvement and team-based training. In this article, we will review the current concepts surrounding project management that are deployed to engage clinical specialists and improve care. Project management requires leadership commitment and an improvement aim. The essential drivers for success include creating an implementation plan, building consensus, developing meaningful data measures, and making continuous improvements.
\end{abstract}

Keywords Complex performance improvement . Interdisciplinary improvement teams $\cdot$ Sustainability and spread

\section{Introduction}

Hospitals are complex organizations that utilize highly trained, autonomously performing clinical professionals. The hospital organizational structure is highly fragmented into decentralized areas (e.g., Emergency Department, Radiology, Catheterization Laboratory, and Operating Room) where care is delivered. Other areas become responsible for supporting efficient patient care processes (e.g., Nutrition, Pharmacy,

F. van Pelt $(\square)$

The Chartis Group LLC, 220 W. Kinzie St., 5th Floor, Chicago,

IL 60654, USA

e-mail: fvanpelt@chartis.com

\section{A. K. Tuli}

Hamad Medical Corporation, Doha, Qatar
Physical Therapy, Laboratory, and Biomedical Engineering). Most often the individuals responsible for managing these areas are previous or current clinicians.

More and more, however, hospitals are now borrowing management practices from other industries. Most facilities are moving toward a customer-focused model or "patient centered" model of care and employing outcome-based goals of treatment. While much attention has been placed on improving electronic information systems, a great deal of effort has turned toward internal process improvement and team-based training. In this article, we will review the current concepts surrounding program management that are integrated to engage clinical specialists and improve care.

\section{Identify and Establish Leadership, Team and Governance Structure}

In order to achieve sustainable results, it is critical to identify leaders that are committed to making the initiative visible and who will guide and support the team in overcoming implementation obstacles as they arise. They are typically responsible for a number of different initiatives; thus, it is also helpful to have clarity around the specific role that leaders will take. In many cases, it is helpful to have leaders serve in a sponsorship role, where the primary responsibility is engagement in strategic planning and supporting a specific operational team that is responsible for implementation. Since performance improvement initiatives take place at multiple organizational levels, it is important to identify leadership within the sponsorship and operational roles at every level where the work is taking place (Fig. 1).

It is also very important to build and enable effective improvement teams (Fig. 2). The most important element of a functional team is its interdisciplinary team composition, with 


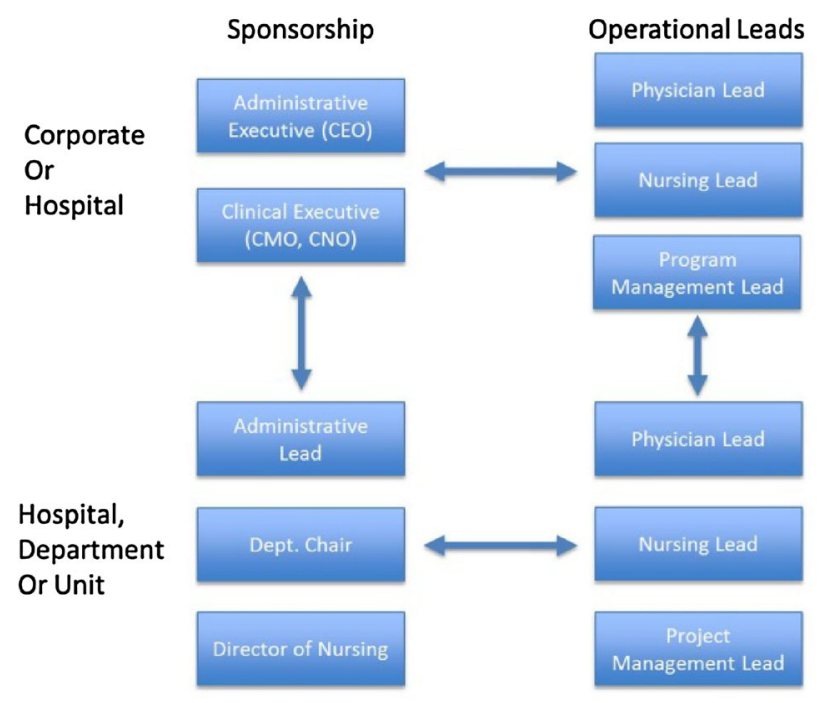

Fig. 1 Leadership roles in project management

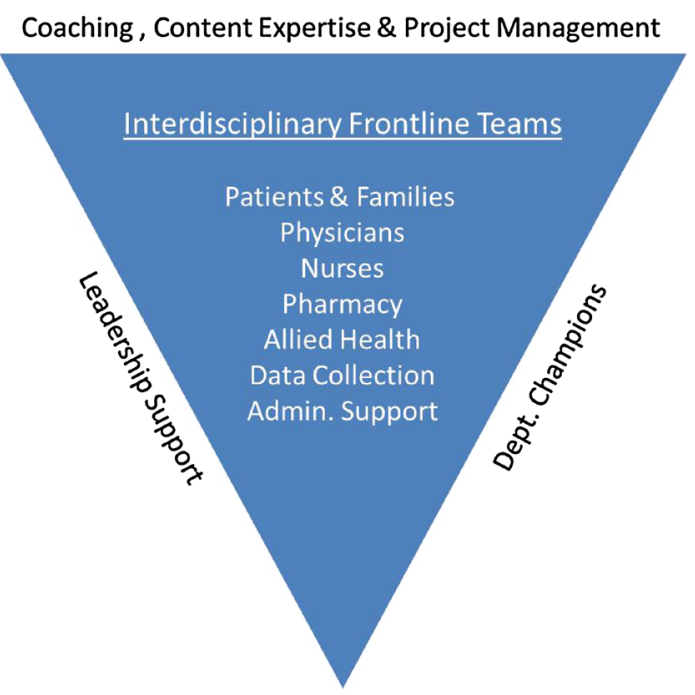

Fig. 2 Building effective improvement teams

cross-sectional representation of staff working in the clinical areas. These interdisciplinary frontline teams may include physicians, nurses, appropriate allied health professionals, representatives from data collection, and administrative support. These interdisciplinary teams generally provide clinical care to patients as their primary function, and it is essential to provide these teams with a support infrastructure that facilitates the extra work that is being asked of them in the context of performance improvement. Leadership support is essential to reinforce the organization's commitment to the program and to provide teams with support while holding all involved accountable to the work. Clinical department champions provide the necessary credibility for the program implementation and serve as content experts and role models during the initial implementation phases. Coaching, content expertise, and project management provide the team with necessary support and guidance throughout the implementation, enabling unit staff to focus on patient care interface [1•]. It is essential for all of these groups to be in close communication with each other and to understand each other's roles and define responsibilities and accountabilities.

Since complex initiatives span multiple levels of the organization, it can be helpful to develop a "line of sight" (Fig. 3), which identifies the progression of guidance and support from senior leadership to the front lines. Leaders and managers at every level should understand their positions within the line of sight and their expected involvement in achieving a successful result. The visible connectivity optimizes channels of communication and opportunities for resolving initiative challenges as they arise.

\section{Understand the Opportunities and Challenges}

Most large-scale improvement initiatives have complex underpinnings that must be identified, quantified, and qualified. Understanding the opportunities and the challenges requires the inputs from all of the involved or affected stakeholder groups. It is important to differentiate between the "formal" process, how a process is designed to work in principle, and the "informal" process, how a process actually works. If improvement initiatives focus on the formal process without understanding and improving the informal process, there is a high likelihood that the improvement will not be sustained and will revert to the original baseline.

There are a number of approaches to identifying the underlying issues. The multidisciplinary team can engage in a brainstorming exercise that initially encourages the listing of any issues considered to be important. By enabling team members to share all of their concerns, this exercise not only exposes critical elements that might not have been otherwise considered but also provides what for many may be their first opportunity to have their concerns heard; this can serve as a powerful means to create team ownership for the improvement initiative. The outputs of the brainstorm are then reviewed and categorized by the team (Fig. 4). These categories then serve as the framework under which important elements are listed to generate a strategic and tactical implementation plan.

\section{Define the Improvement Aim and the Essential Drivers for Success}

In keeping with the leadership's "line of sight" that provides direction and accountability to the implementation 
Fig. 3 Project line of sight

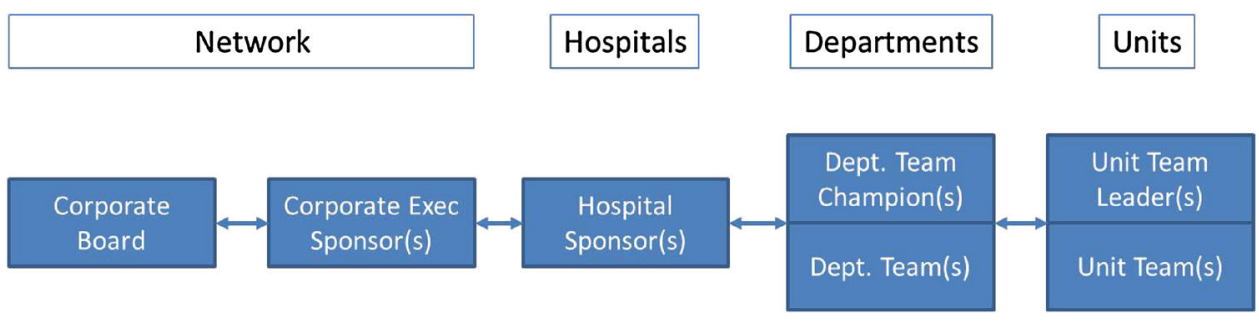

process, a driver diagram (Fig. 5) can serve to provide a meaningful content interface to the stakeholders at all levels of the organization [2•]. The aim statement describes the overall objective or desired outcome of the improvement initiative. Not only does it offer a concise description of the goal, but also provides a clear measurable outcome and a timeline by which the defined objective should be achieved. The primary drivers describe the major components that must be successfully implemented in order to achieve the aim. These primary drivers are supported by secondary drivers that in turn describe the important elements that must be achieved in order to fully implement the primary drivers. Once the secondary drivers have been identified, the next level of detail generally begins to describe specific activities that must be undertaken as part of the implementation.

The completed driver diagram provides all levels of the organization with a comprehensive overview of the improvement initiative. All levels are able to appreciate how the specific activities connect and support the successive progression of imperatives that must be in place to achieve the improvement aim. This again reinforces the necessary sense of ownership as well as the roles, responsibilities, and accountability at all levels of the organization.

\section{Create an Implementation Plan with Timeline}

The driver diagram provides a template from which to develop an implementation plan and timeline. Most complex improvement initiatives benefit from having a detailed implementation plan and timeline that provides a clear direction with transparency around roles, responsibilities, activities, and expected outcomes. While this may be perceived to be a time-consuming exercise that detracts from initiating the work, in the long run it will reduce the amount of re-work that frequently occurs when projects are implemented in haste. In addition to identifying the project components, activities, and milestones, it is important to identify in advance the potential risks and critical decision points that can impact the success of the initiative. Having measurable outcomes and, importantly, baseline measures that reflect success of the improvement initiative serve to garner ongoing support and ownership around the improvement. Identifying by name those individuals who will be actively engaged with the initiative, as well as where and for how long the various activities will take place, enhances accountability and the ability to achieve expected milestones. Although it is inevitable that implementation plans will undergo continual adjustment as additional project opportunities and challenges are identified, the initial plan and timeline will serve as a solid foundation.

Recognizing that there will be a large amount of activity that takes place by the implementation teams at the point of care, it is helpful to create planned convergence periods for teams to debrief and to develop or refine implementation plans and activities (Fig. 6). These convergence sessions may take the form of workshops and can be structured to include internal and/or external content experts, program managers, and leadership to affirm commitment to guidance and support for the teams. These sessions are most effective when facilitated and interactive, and should focus on critical implementation elements such as the primary and secondary drivers. If these sessions are structured to enable the exchange of team learning, and provide clear objectives with expected outputs that provide teams with defined action items for the next interim work period, the teams usually come away empowered to meet their next set of objectives. The workshops are generally front loaded during the early stages of the initiative at six to eight-week intervals, and continue in frequency until the major implementation elements have been addressed or until the teams have established a consistent and functional methodology of implementation within a test unit.

\section{Building Alignment \& Ownership}

A common barrier to engaging improvement teams and achieving sustained improvement is when leadership delivers a directive mandate for change (Fig. 7). In contrast to the logical progression of problem solving that begins with understanding the issues, generating a differential list of solutions, and ultimately selecting the best alternative for implementation, it is common to apply this process in reverse $[3 \bullet, 4 \bullet]$. While well intended and seemingly more 


\begin{tabular}{|c|c|c|c|c|c|c|c|c|c|}
\hline$\frac{5}{8}$ & 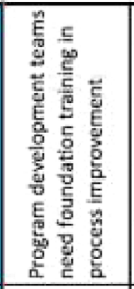 & 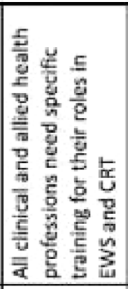 & 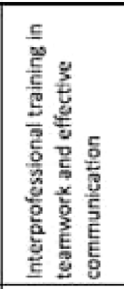 & 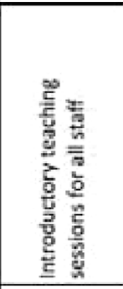 & 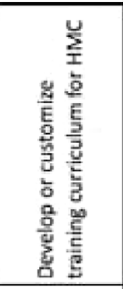 & 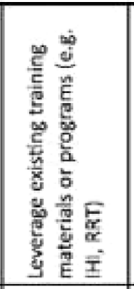 & 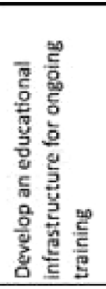 & & \\
\hline 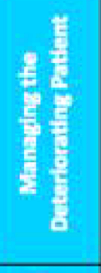 & 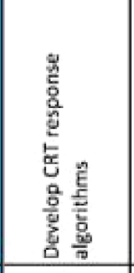 & 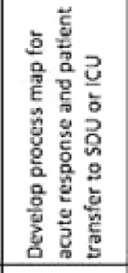 & & & & & & & \\
\hline 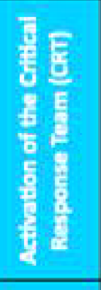 & 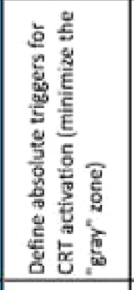 & 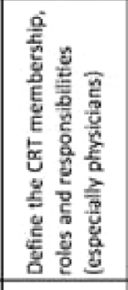 & 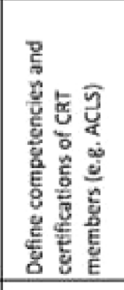 & 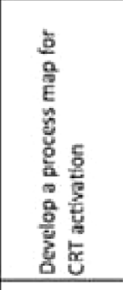 & 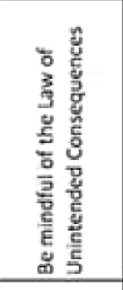 & & & & \\
\hline $\begin{array}{l}\frac{5}{8} \\
\frac{5}{5} \\
\frac{5}{5}\end{array}$ & 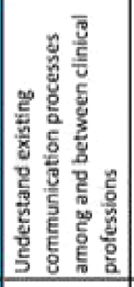 & 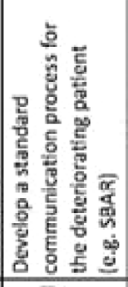 & 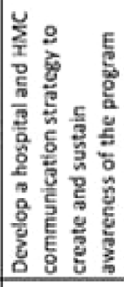 & & & & & & \\
\hline 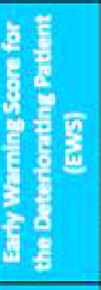 & 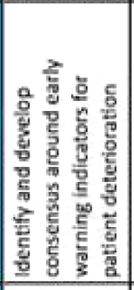 & 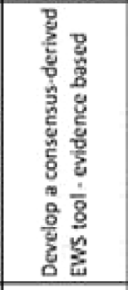 & 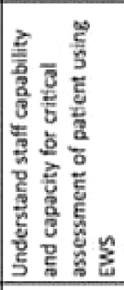 & 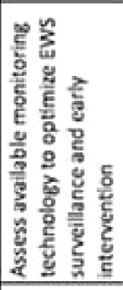 & 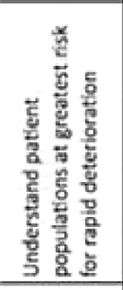 & 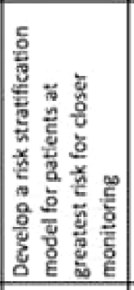 & 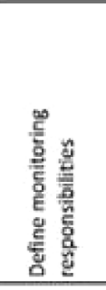 & 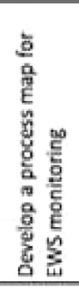 & 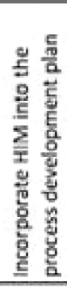 \\
\hline 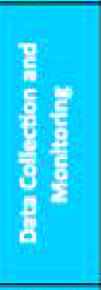 & 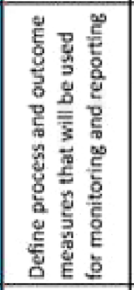 & 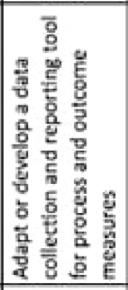 & 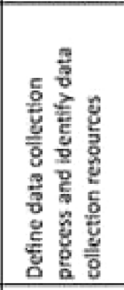 & 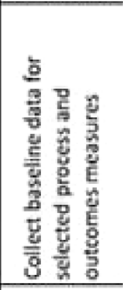 & 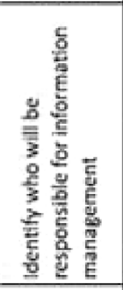 & 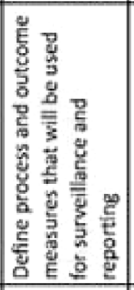 & & & \\
\hline 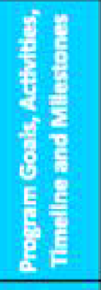 & 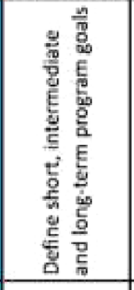 & 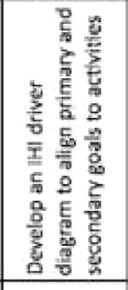 & 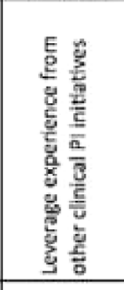 & 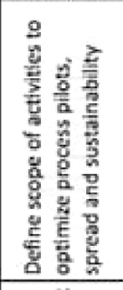 & 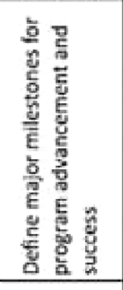 & 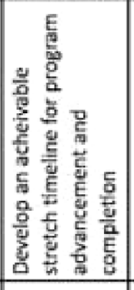 & & & \\
\hline 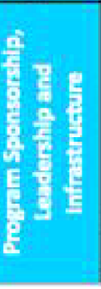 & 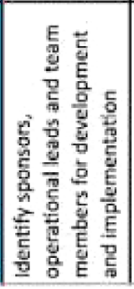 & 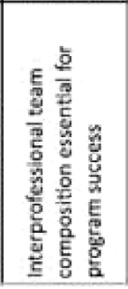 & 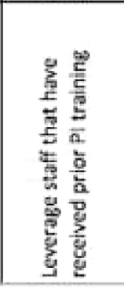 & 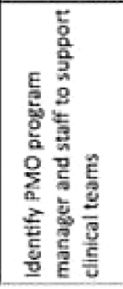 & 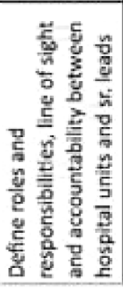 & 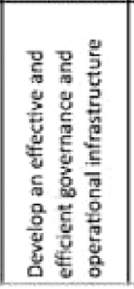 & & & \\
\hline
\end{tabular}


Fig. 5 Driver diagram

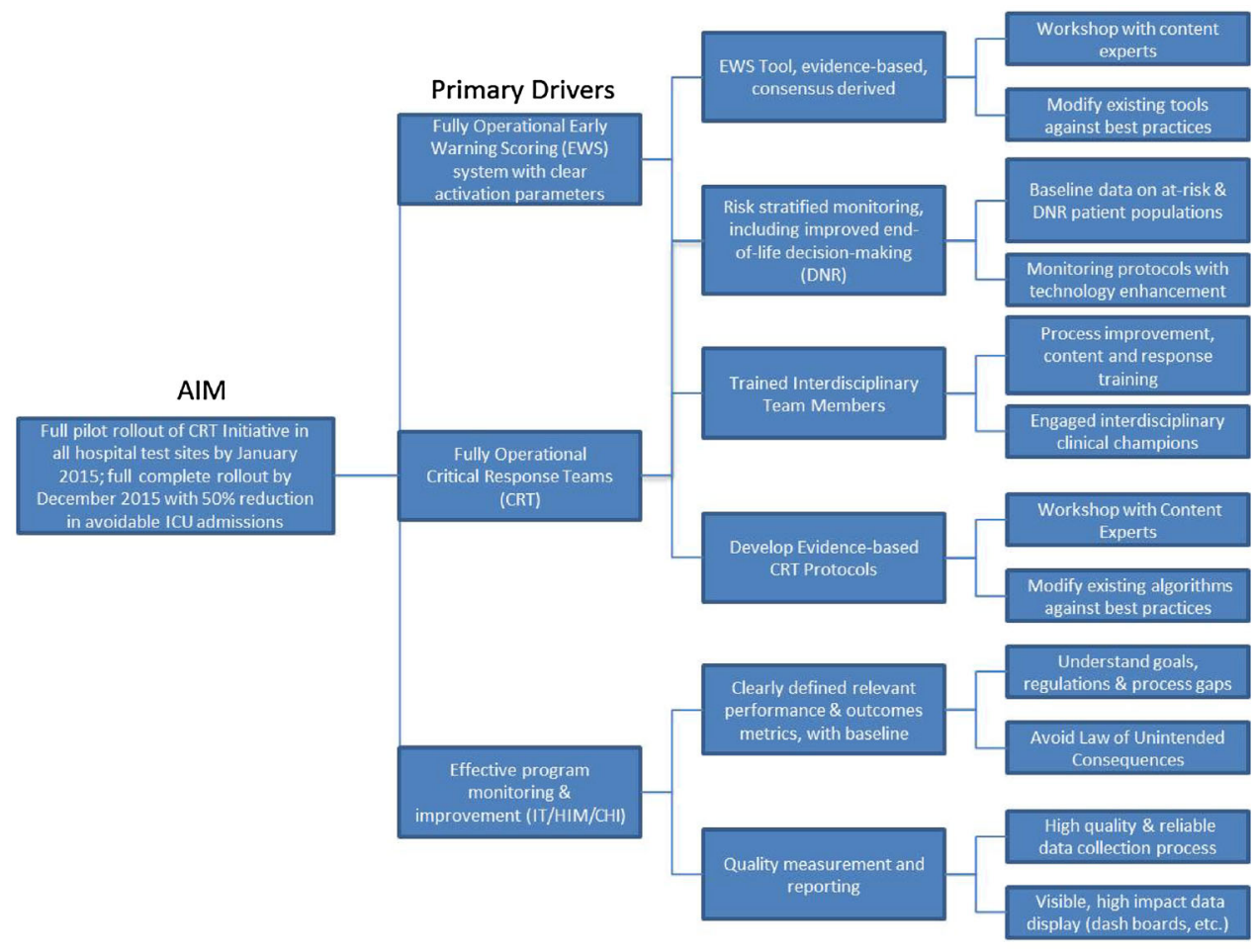

efficient to provide improvement teams with a comprehensive solution upfront, this approach often fails to include the vital inputs of frontline professionals, resulting in a suboptimal solution, lack of ownership, and ultimately an unsustainable improvement [5•]. Leadership should recognize that sharing a specific strategic need with improvement teams is best executed when the teams can develop the implementation plan based on the local context of the care setting.

\section{Enable Relevant Process Improvement}

In order to optimize the successful and sustained implementation of a complex performance improvement initiative, it is important for leadership to provide a clear objective across the organization (Fig. 8). This objective, the "What," should be consistent and aligned with the driver diagram, and should have an associated "line of sight" to clarify leadership accountability for initiative implementation. The implementation, the "How," should be developed by the improvement teams based on local context and taking into consideration the specific service nuances that exist at and within each level. This again ensures relevance and ownership of the improvement by the users. It is important to recognize that every level in an organization has an element of the "what" and the "how" associated with it all the way to the unit level. Although there will likely be process adjustments that necessarily reflect service differences across and within levels, as long as the "what" remains consistent with the driver diagram levels, the implementation at every level should be aligned.

In addition to recognizing the importance of the "what" and the "how," it is also critical to identify demonstration sites where process improvements can be efficiently tested before organization-wide implementation occurs. This approach enables rapid improvement test cycles to be utilized in a controlled environment where outcomes are contained. As the improvements are refined and the results become evident, they can be formalized and shared with other areas as a foundation for further rollout.

\section{Develop Meaningful Measures and Standardize Data Collection}

Developing measures that actually reflect the intended improvement requires careful attention. There are many examples where developed measures follow the "Law of Unintended Consequences" and create an undesired result. For example, defining the measure for on-time operating room (OR) starts as the time a patient enters the OR can result in patient preparation that would otherwise have occurred in the preoperative holding area now taking place in the OR; while the measure suggests that very little improvement has actually occurred. Process measures can 


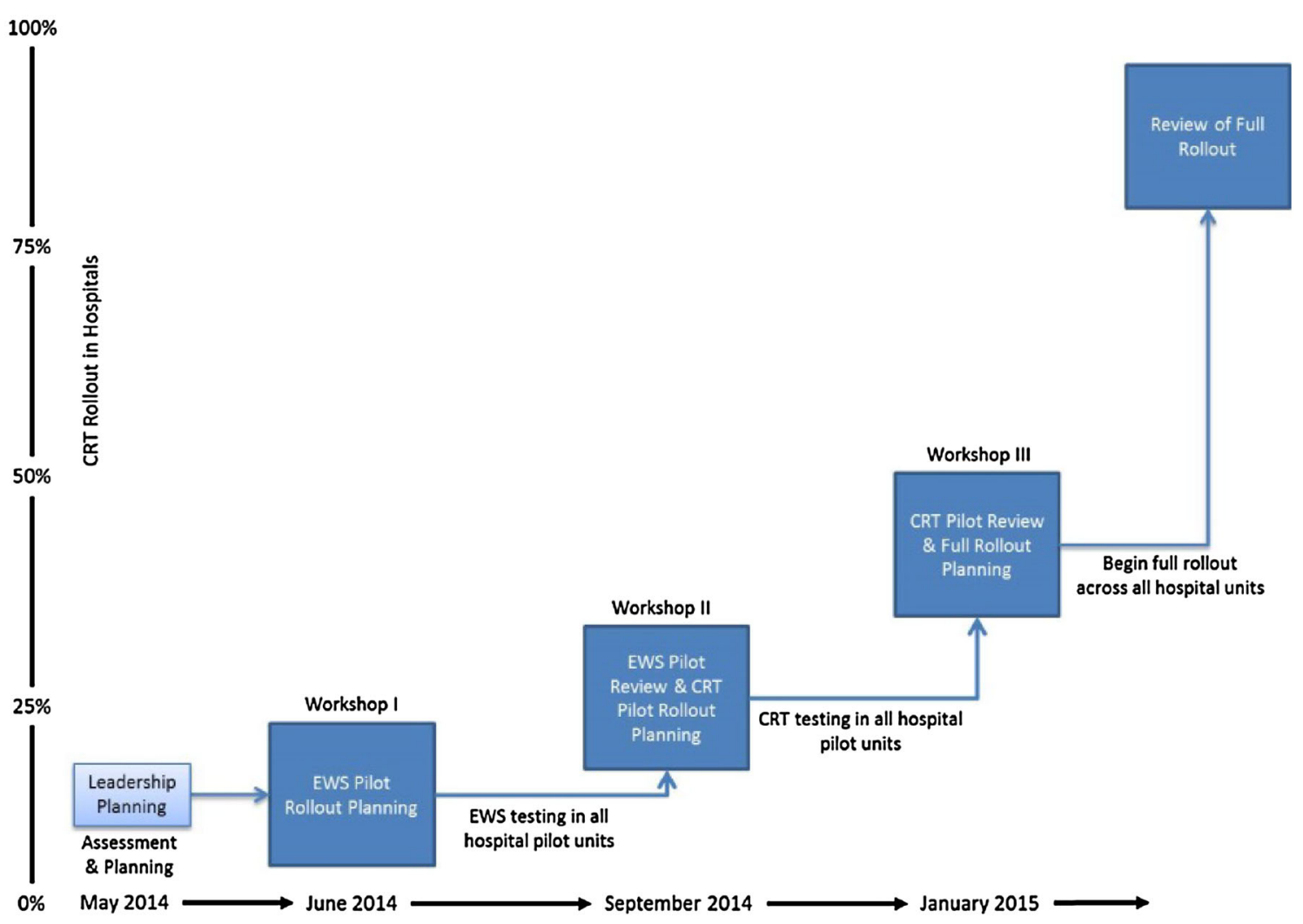

Fig. 6 Convergence periods

be as important as outcome measures and the relevance of the measures can evolve as the performance improvement initiative matures.

Unlike the adjustments to the implementation process that may be appropriate at and within the various levels of an organization, it is critical for measurement and data collection to be standardized across the organization. It is very difficult to evaluate and to report improvement accurately unless measure definitions, data collection methods, and analytics are consistent. While organizational standards must be established across the organization, measures and data must also be relevant and useful at all levels of the organization; one type of report is not likely to fit all audiences. Accordingly, data reporting should be customized as appropriate so that it can be translated into actionable outputs.

\section{Make the Initiative Visible}

In the current healthcare environment where numerous performance improvement initiatives take place in the midst of busy patient care demands, it is helpful to develop a communication plan that makes the initiative visible and aligned. The initiative needs to attract the attention of the organization from clinical and administrative leadership to frontline professionals in a manner that emphasizes the importance, the commitment, the support, and the expected outcome. It will likely be necessary to create a variety of communication venues that reach different audiences with customized relevant messaging. These may take the form of a launch event to senior leadership that includes messages from senior initiative sponsors and nationally or internationally recognized content experts, grand rounds, department presentations, webinars, and email. Emphasizing the alignment of the improvement initiative via the driver diagram to the organization's strategic plan and to other projects underway is helpful to reduce improvement fatigue and to convey relevance.

\section{Celebrate Success}

It is easy to overlook the effort and the commitment required to bring performance initiatives successfully to completion. In addition to the time commitment involved, performance improvement requires a willingness to change work patterns and behavior, which can be difficult. 

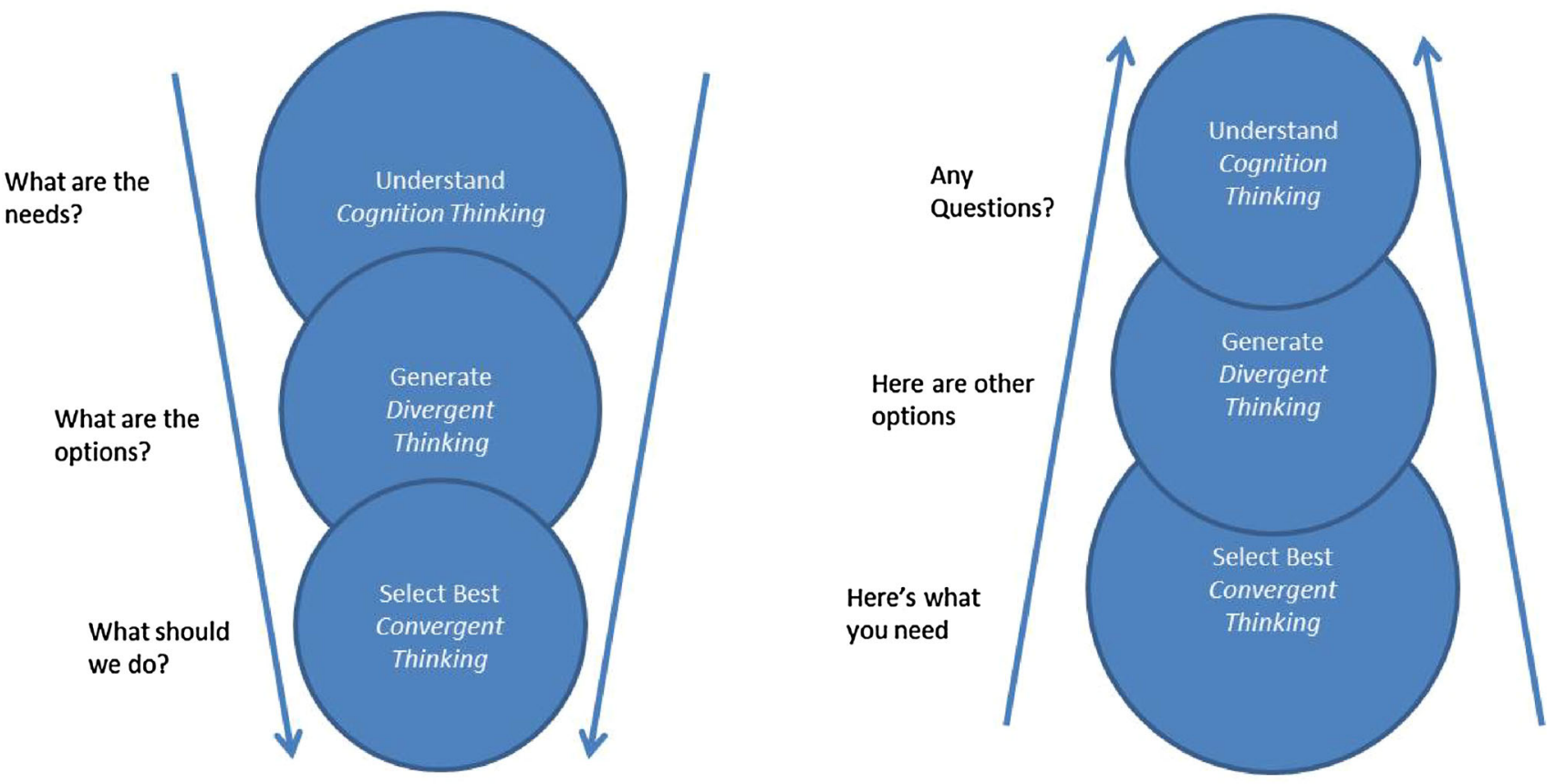

Miller Heiman

Fig. 7 Alignment and ownership for change
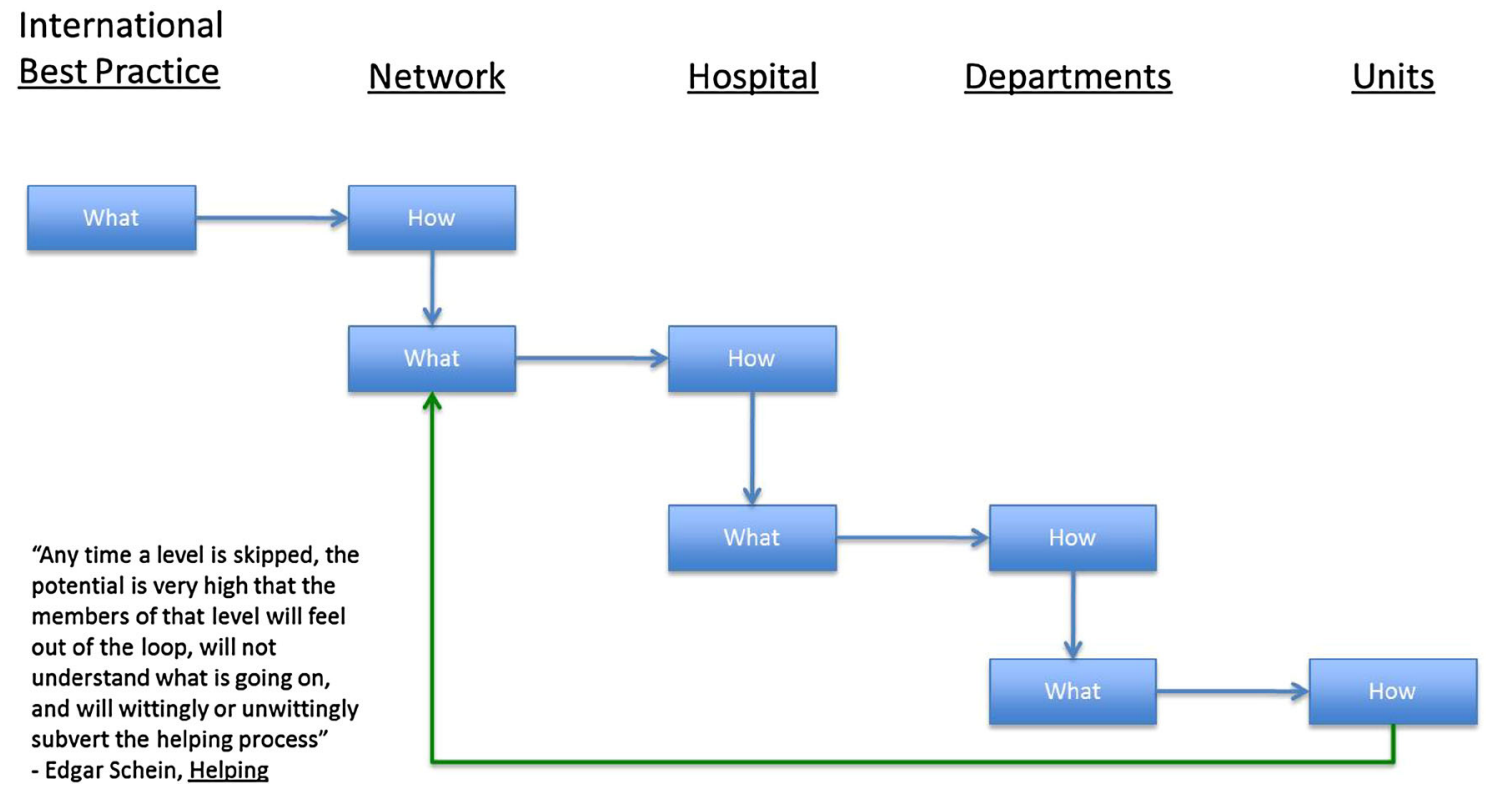

Fig. 8 Alignment and implementation

Creating opportunities to recognize the work being done and to celebrate the successes being achieved validates the efforts and can instill a sense of satisfaction with the accomplishment, while affirming the value of the team- based improvement approach. Recognizing success can take different forms including verbal acknowledgment, presentations, publications, certificates of recognition, gift certificates, and celebrations. 


\section{Case Example: The Early Warning Scoring System (EWS) and Critical Response Team (CRT) Initiative: An Illustration of the Performance Improvement Principles Applied}

The early identification and care intervention for hospital inpatients whose hemodynamic status is rapidly deteriorating has received increasing attention as a quality and patient safety issue. Many organizations are developing an organized response that includes an early warning scoring system (EWS) designed to trigger the activation of a critical response team (CRT). Ideally, this system will capture patients in early reversible stages of decompensation that would otherwise lead to conditions such as sepsis or myocardial infarction, thereby reducing the likelihood of these patients having to be transferred to higher acuity care such as intensive care units. We will use an organization's objective to develop and implement an EWS/CRT program to illustrate the application of these nine performance improvement steps.

After senior leadership had identified the EWS/CRT initiative as a top priority within their quality and patient safety strategy, two leadership teams were created to provide sponsorship and operational guidance and support, respectively. The initiative would require significant participation of physicians and nurses, and the sponsoring leadership team included the Chief Medical Officer (CMO), the Chief Nursing Officer (CNO), and the Vice President for Quality and Patient Safety; the operating leadership team included the physician director of the hospitalist program, the director of critical care nursing, and a program manager from the performance improvement group. Recognizing that the initiative would require a similar structure of sponsorship and support at the department level, a complimentary team of sponsors and operational leads was identified to provide closer support and guidance to the implementation teams.

Interdisciplinary teams were assembled that included representatives of stakeholders that would be involved in the operation of the EWS/CRT. Members included a patient and family representative, physicians, nurses, pharmacists, allied health professionals, data collection specialists, and administrative support. These teams would focus on the clinically relevant elements of the implementation and were supported by leadership, a respected physician champion, and a team with clinical content expertise and experience with the implementation of complex improvement initiatives.

In order to optimize communication, support, and guidance, a reporting structure was established from the unit level to the corporate board of directors. This line of sight provided clarity around roles, responsibilities, and accountabilities expected at every level of the organization for the initiative to succeed.

With the infrastructure and governance structures defined, the operational leads conducted a brainstorming session with the implementation team members. All of the members were encouraged to share their perspectives on the potential implementation issues that would need to be addressed. A series of categories were distilled from the issues, which were then transposed into a grid and served as a means for the team to refine critical implementation elements. For example, under the EWS scoring category, among the six initial issues identified was the need to identify the patient populations at greatest risk for deterioration, and the need to develop by consensus an evidencebased set of EWS indicators.

A driver diagram was created using the EWS/CRT issues grid. The aim of the initiative was to establish pilot test sites in selected care units within six months and to progress to a complete implementation with a $50 \%$ reduction of preventable ICU admissions within one and a half years. Three primary drivers were identified as critical elements to achieve the aim, one of which was the development of a fully operational CRT team. These primary drivers would be dependent on the successful attainment of six secondary drivers, which in turn would require a number of action items to complete. The completed driver diagram provided the leadership, managers, and teams a comprehensive understanding of the integrated nature of the initiative, connecting specific activities to the higher aim through all levels.

The completed EWS/CRT driver diagram served as a foundation to develop a detailed implementation plan and timeline. A series of workshops targeting the primary and secondary drivers were planned within the initiative timeline to bring implementation teams together around objectives and action items required to attain these milestones. The interim work periods for the teams between the workshops would be supported by the operational leads at the various levels to optimize successful completion of the action items, in preparation for the next workshop and subsequent set of activities. As the teams developed their processes and became increasingly selfsufficient, the frequency of the workshops would diminish and implementation team members would increasingly assume leadership and educational roles to their colleagues as implementation progressed across the organization.

Given the nuances in the patient populations and the care processes between care units, it was clear that the EWS/CRT implementation would require a contextual adjustment. Similar to the approach used during the development of the issues grid, the driver diagram and the 
workshop curriculum, an inclusive team approach would be used to translate strategic goals into tactical implementation. For example, an early workshop focused on the need to develop an effective EWS tool. Rather than insisting that the organization adopts an already developed template, teams would be encouraged to use a best practice example and to modify the scoring system to reflect the local patient conditions and care approaches. Not only would this create a more relevant EWS tool, but would also create a sense of ownership for the tool that would not otherwise have happened. As teams reconvened at subsequent workshops and shared their learning and implementation approaches, the teams would be able, through skillful facilitation by content experts, to reach consensus around a single consolidated EWS form. By presenting the larger objective (the What) and enabling teams to determine the implementation (the How), the organization would achieve a result consistent with the higher strategic imperative.

Although the operational leadership would encourage an expanded approach to implementation and would tolerate a certain degree of process variation, the insistence on utilizing a consistent set of defined measures, data collection, and analytic approaches would be unwavering. At a process level, EWS scores that triggered a CRT response would be consistent across every unit. At an outcome level, the measure of a successful CRT intervention would focus on the number of avoidable ICU admissions. This would create the ability to compare initiative efficacy across the organization and enabled unit challenges and best practices to be shared with relative uniformity.

The organization had a number of complex priority initiatives underway simultaneously, and it would be important to maintain focus on the progress being made with EWS/CRT. At the outset of the initiative, a launch event would have been held for senior administrative and clinical leadership, which would include a keynote address by an internationally recognized expert on the topic. This launch event would be followed by a grand rounds session for clinical staff as well as departmental presentations to nursing and medical-surgical specialties. Implementation teams would receive prepared materials from the operational leadership teams to provide ongoing focused educational sessions, and mandatory sessions for residents on the EWS/CRT initiative would be held through the department of medical education. Periodic letters from leadership sponsors to leadership colleagues maintained the visibility at senior levels, and scheduled email updates to organizational staff would keep everyone informed. Data displays in the form of run charts and dashboards would also serve as an important communication tool for the significant improvements being made. The results would be reported to leaders and to the medical executive committee, which would enable action items to be generated in response with accountable follow-up. The communication plan was structured and deliberate, leveraging the established governance structure as well as the defined line of sight.

Concerted effort would be made to acknowledge the work being done when significant progress was made or initiative milestones were reached. Some teams would celebrate locally, while other recognition events would take place as a collective. Certificates of recognition were particularly popular within the organization, particularly if presented by the sponsoring leadership. These instances of recognition and celebration would validate the extra effort that teams were committing to initiatives that extended beyond their usual work obligations and would reinforce the value of their contribution to improving the organization and the quality and safety of patient care.

\section{Summary}

There are many performance improvements and patient safety initiatives underway in healthcare that utilizes a variety of described approaches including Lean, Six Sigma, Kaizen, Toyota Quality Management, and Microsystems. The challenge common to all approaches is to engage an organization's leadership, administrative and clinical staff in an integrated manner such that improvement initiatives are aligned with the organization's strategic goals and can be transposed from concept to sustained implementation. In this article, we have identified nine important areas that must be carefully addressed to achieve a sustained, measurable, and meaningful improvement:

Identify and Establish Leadership, Team, and Governance Structure

Understand the Opportunities and Challenges

Define the Improvement Aim and the Essential Drivers for Success

Create an Implementation Plan with Timeline

Building Alignment and Ownership

Enable Relevant Process Improvement

Develop Meaningful Measures and Standardize Data

Collection

Make the Initiative Visible

Celebrate Success

The case study for the planning and implementation of an Early Warning System and Critical Response Team (EWS/CRT) initiative illustrated the application of these 
principles. It is clear that there are many additional areas of detail that could be described in and around these concepts. This article can also provide the framework for those more detailed discussions.

\section{Compliance with Ethics Guidelines}

Conflict of Interest Frederick van Pelt and Alejandro Kohn Tuli report no conflicts of interest.

Human and Animal Rights and Informed Consent This article does not contain any studies with human or animal subjects performed by any of the authors.

\section{References}

Recently published papers of particular interest have been highlighted as:

- Of importance
1. - Nelson CN, Batalden PB, Godfrey MM. Quality by Design. San Francisco: Jossey-Bass; 2007. This is a textbook that describes the foundation of performance improvement at the frontline of care.

2. - Institute for Healthcare Improvement 90-Day Research and Development Process. Available at: http://www.ihi.org/about/ Documents/IHI90DayResearchandDevelopmentProcessAug10. pdf. This is an article that describes the value of creating driver diagrams to provide an aligned overview of critical performance improvement elements.

3. - Miller RB, Heiman SE. The New Conceptual Selling. 3rd rev. ed ed. New York: Grand Central; 2005. p. 37-47. This book describes the importance of a conceptual framework for performance improvement that combines advisory expertise with local context. While it is a book focused on sales, the principles of creating a desired outcome through collaboration are very relevant in clinical improvement initiatives.

4. • Lipmanowicz H. "Buy-in" versus "Ownership." Available at: http://www.liberatingstructures.com/storage/articles/henri-1/BuyIn\%20v.\%20Ownership.pdf. This article offers a distinct between getting people to accept one's own idea versus framing it is their own.

5. - Schein E. Helping. San Francisco: Barrett-Koehler; 2009. 141 p. This book focuses on the value of humble inquiry, which emphasizes the importance of active listening as a means of understanding the needs of those seeking one's help. 